\title{
Image sensors for digital photography: a short course for undergraduates. I: Optics
}

\section{Sensores de imagen para fotografía digital: un minicurso para estudiantes universitarios. I: Óptica}

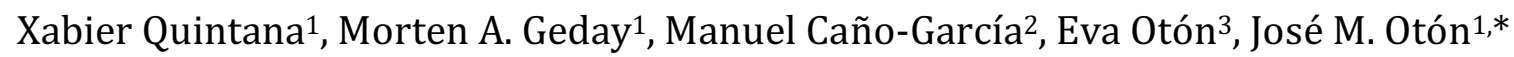

1. CEMDATIC, ETSI Telecomunicación, Universidad Politécnica de Madrid, Av. Complutense 30, 28040 Madrid, Spain

2. INL International Iberian Nanotechnology Laboratory, Av. Mestre José Veiga s/n, 4715-330 Braga, Portugal

3. Institute of Applied Physics, Wojskowa Akademia Techniczna, ul. gen. Sylwestra Kaliskiego 2, 00-908 Warszawa, Poland

*e-mail: jm.oton@upm.es

Received: 28/02/2020 Accepted: 24/03/2020

DOI: 10.7149/OPA.53.1.51040

\begin{abstract}
:
Over the last half century, photography has evolved from photochemical reactions induced on a photosensitive film to optoelectronic devices employed in digital image. Video has done the same migration from classical film movies to optoelectronic acquisition and storage. Digital and video cameras share optics with traditional film cameras, but the image recording is realized by an optoelectronic device -a CCD or CMOS sensor- rather than a film. The quantity of digital photo and video images has dramatically increased in the last decade, arising from the ubiquitous cameras of mobile phones. Despite their popularity, condensed tutorial articles on the subject are still scarce. This work intends to contribute to the knowledge of this field with a medium-level short course that is currently being taught in a broader subject (Consumer Photonics) to undergraduate students of Telecommunication Engineers at the Universidad Politécnica de Madrid. The course can be followed by students having a basic background on Physics and specifically on Optics and Semiconductors. The course is divided into two chapters. An introduction to optics of cameras and basic concepts of photography has been considered necessary to follow the second part, which is entirely devoted to optoelectronic sensors.
\end{abstract}

Key words:

Digital imaging, CMOS, CCD, optoelectronic sensor

\section{REFERENCES AND LINKS}

[1] Robert Hirsch, "Seizing the Light: A Social \& Aesthetic History of Photography". 3rd edition, New York, Routledge (2017) pp.11-15.

[2] José M. Otón, "Móviles plegables, pantallas que se enrollan... solo hace falta mejorarlos" The Conversation http://theconversation.com/moviles-plegables-pantallas-que-se-enrollan-solo-hacefalta-mejorarlos-112926 (2019)

[3] Roger N. Clark, ClarkVision.com, "Notes on the Resolution and Other Details of the Human Eye" https://clarkvision.com/imagedetail/eye-resolution.html (2020).

[4] John P. Frisby, "Seeing: Illusion, Brain, and Mind". Oxford University Press, Oxford (1980) 
[5] Photography, "How does photography (as opposed to physics) define 'focal-plane'?" https://photo.stackexchange.com/questions/45970/how-does-photography-as-opposed-to-physicsdefine-focal-plane (2020)

[6] Dan Cart, "How to read an MTF chart". Shutter Muse, https://shuttermuse.com/how-to-read-mtfchart/ (2020)

[7] Rubén Osuna, "Do sensors "outresolve" lenses' capabilities?". Universidad Nacional de Educación a Distancia, Spain, https://www2.uned.es/personal/rosuna/resources/photography/Diffraction/ Do\%20sensors\%20outresolve.pdf (2020)

[8] Wikipedia, "Zeiss formula". https://en.wikipedia.org/wiki/Zeiss_formula (2020)

[9] René Bouillot, “Curso de fotografía digital”, Ed. Omega S.A. (2005), ISBN 84-282-1390-9

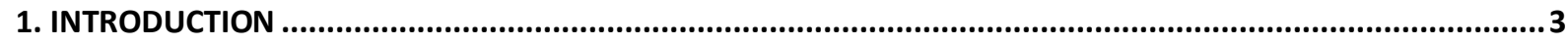

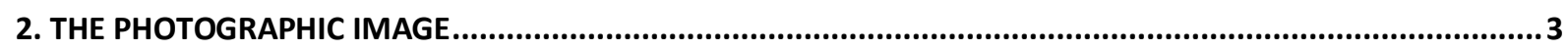

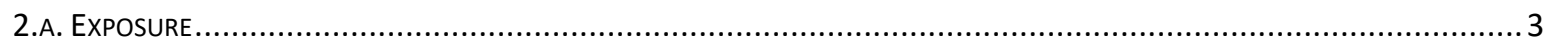

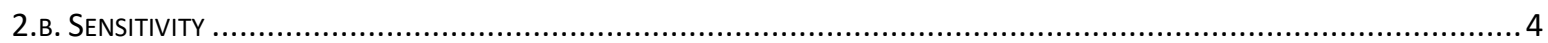

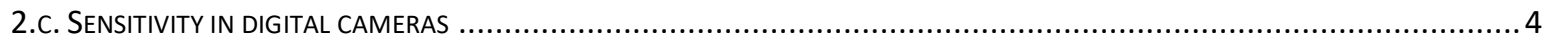

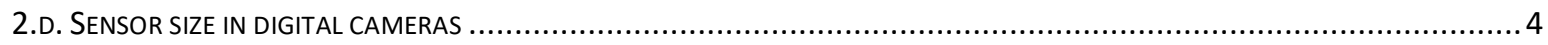

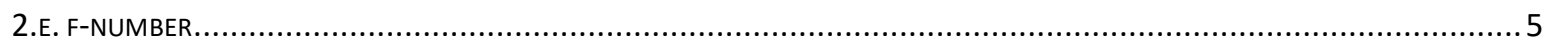

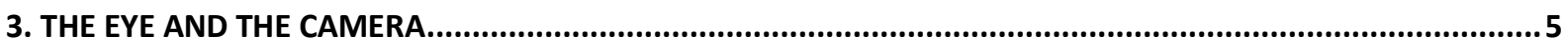

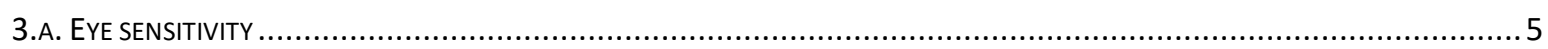

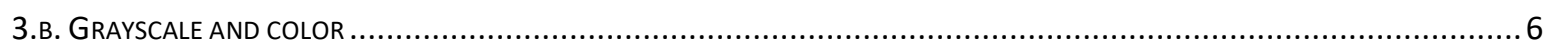

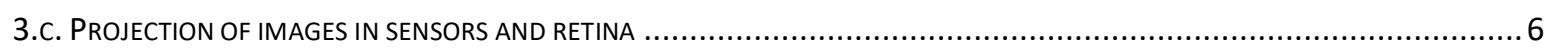

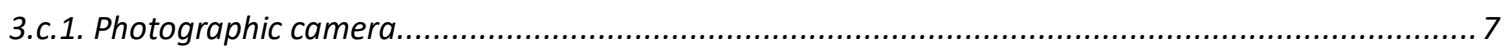

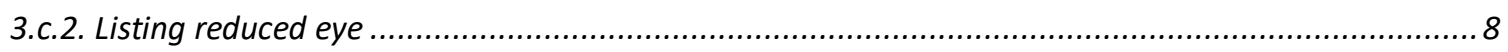

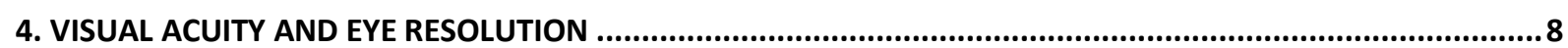

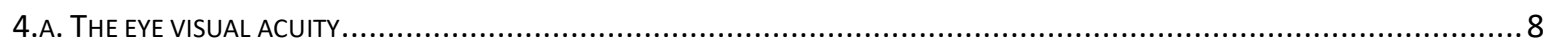

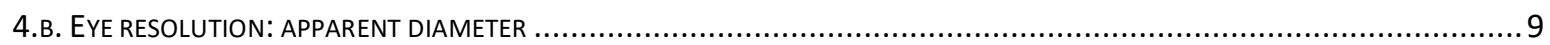

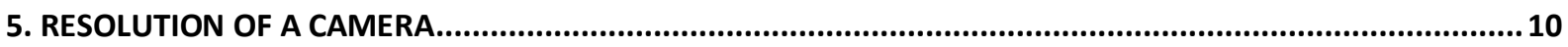

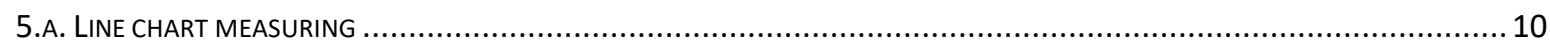

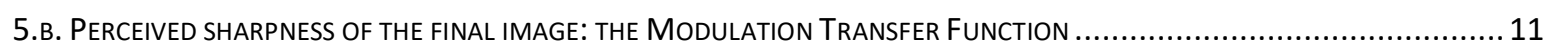

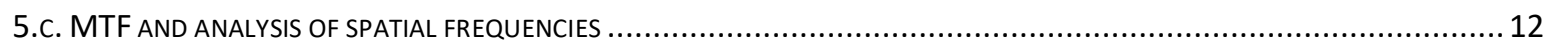

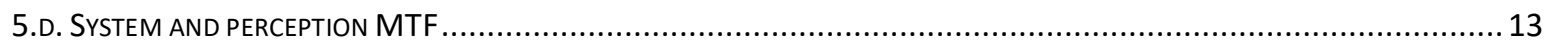

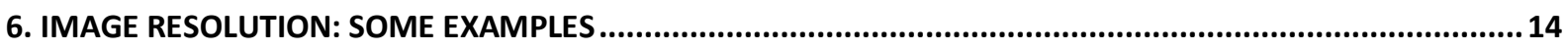

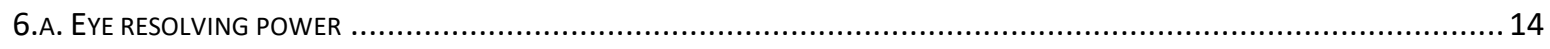

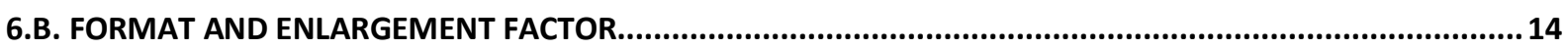

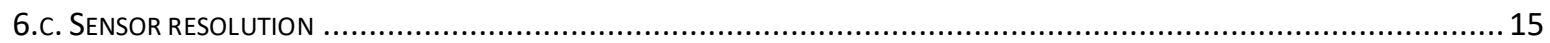

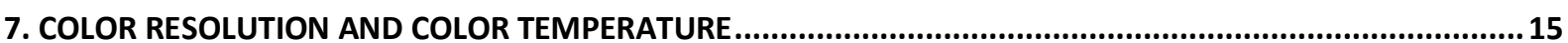

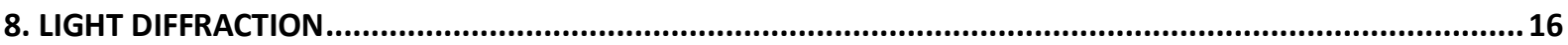

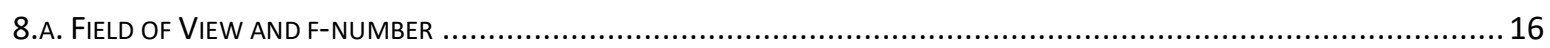

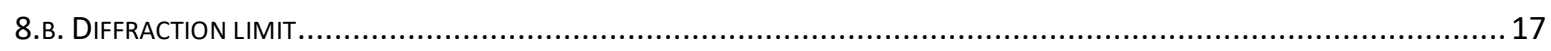

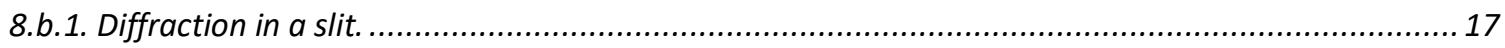

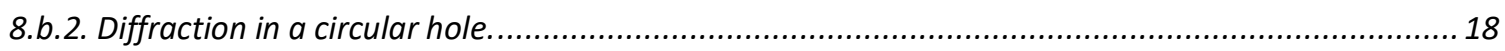

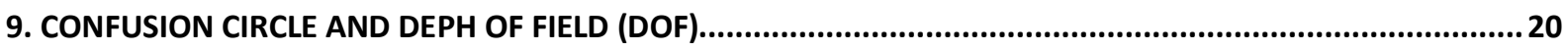

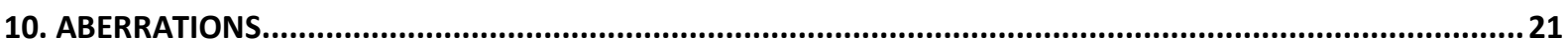

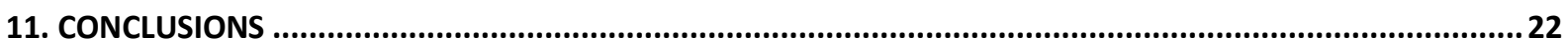




\section{Introduction}

Photography is nearly two centuries old since the first crude attempts of Nicéphore Niépce by mid-1820s, and the invention by Louis Daguerre of the daguerreotype process [1] in the following decade. With time, the process remarkably improved -sensitivity, cinema, color, resolution- but its fundamentals remained substantially the same, a photosensitive emulsion capturing the image, eventually transferred to a paper support or a celluloid in movie films. In the last quarter of XX century, alternative acquisition and storage methods were developed. Nevertheless, the huge increase of photography captured by electronic means originated in the first decade of this century, in parallel to the development of smartphones. This revolutionary disruption has effectively doomed old film cameras to become museum specimens.

The optical features of digital cameras are quite coincident with traditional film cameras, but the image recording is realized by a CCD or CMOS sensor -the second being preferred nowadays- rather than a film. This new image handling has brought two important side effects: the possibility of taking hundreds or thousands of pictures at literally no cost, and the possibility of obtaining remarkably good pictures out of miniature cameras. Indeed, digital cameras were born and are being developed in the same time frame as mobile phones. Almost all current mobile phones feature a high-resolution, small size digital camera that eventually may wipe out the market of compact cameras. On the contrary, sales of medium and large-size reflex cameras (Single-lens Reflex cameras, SLR) and Electronic Viewfinder with Interchangeable Lens (EVIL) cameras (also called mirrorless) are still steadily increasing among the photography fans, mainly because their functionality is different, and their price is becoming attainable.

In the future, the market trends will possibly lead to a new kind of ultraportable devices including phone, agenda, photo and video cameras, and TV. The size of the display, as commented elsewhere [2], is a big issue in this subject. Anyhow, the quality of the camera will have to improve substantially for the SRL cameras to lose their current market.

This short introductory course on digital cameras has been divided into two sections. The first section reviews the optics of a photographic camera and how optics determine the quality of the final image. The properties of the camera lens are shown and compared to the properties of the human eye. The impact of a number of relevant parameters such as sensor resolution, enlargement factor, diffraction, depth of field, etc. are studied as well. The second section studies the different sensors employed in digital photography, and their behavior as optoelectronic devices.

\section{The photographic image}

For an image to be formed, it is necessary to bring a certain amount of light containing the scene information to a detector, being a retina, a photosensitive emulsion, or an optoelectronic sensor. A correct image is obtained when the amount of light is enough (threshold) but not excessive (saturation) for the detector to store the information. We shall start our discussion studying the three variables that control the correct amount of light: exposure, sensitivity and $f$-number $(f \#)$

\section{2.a. Exposure}

Exposure is the amount of light reaching the eye retina or the camera sensor or film. The mechanisms that determine the amount of light gathered by an eye and a camera are substantially different; still, some

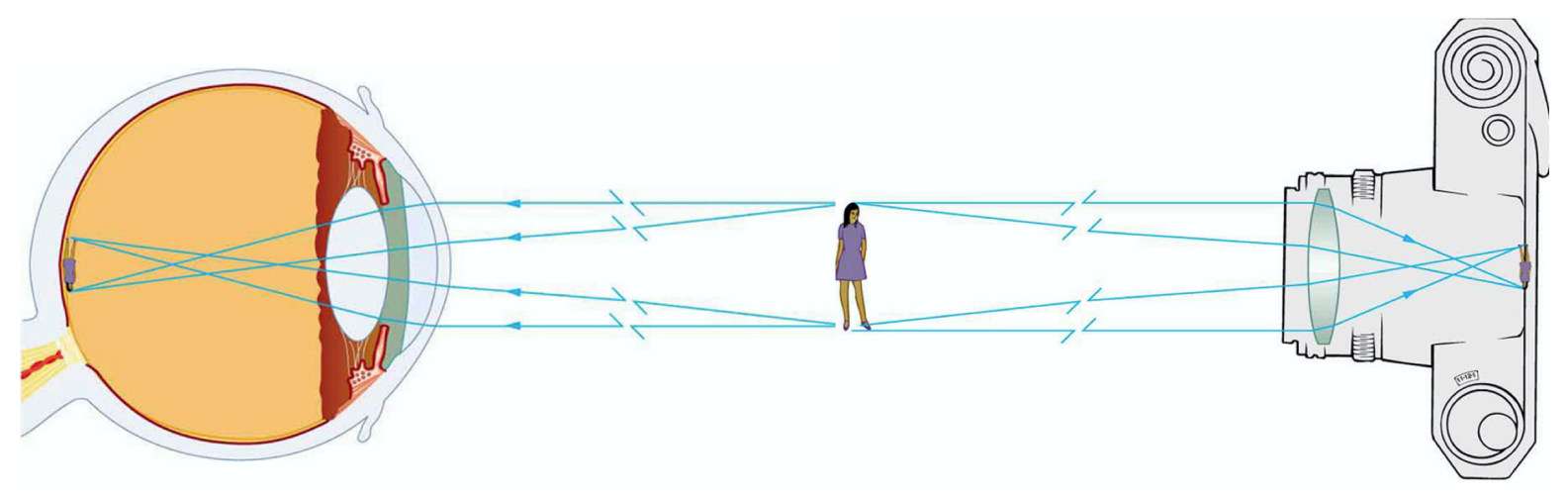

Figure 1. Projection of an image in the retina and in a camera 
similarities may be pointed out, especially in compact cameras (Fig. 1). The light impinging onto a photograph is ultimately determined by two parameters: $f$-number and shutter speed. The f-number, a.k.a. $\boldsymbol{f} \#$ for short, is the ratio between the focal length and the diaphragm aperture. The shutter speed regulates the time the diaphragm is open; it is customarily given in fractions of seconds. A third parameter, the film or sensor sensitivity, also affects the brightness of photographs, although it does not control the amount of received light but rather the effect that light produces on the film or sensor.

\section{2.b. Sensitivity}

The film sensitivity is associated to the grain size: usually the sensitivity is higher in larger grain sizes. For the same f-number, a film having double sensitivity requires half of the time -hence double speed-for the same exposure. Films with high sensitivity allow pictures to be obtained with dim light; however, larger grain sizes make the image noisier. Sensitivity in conventional film-based analog photography is associated with the film itself. Changing the sensitivity obviously implies changing the film. This is not the case in digital photography, whose sensitivity depends on the electronic amplification of the pixel signal.

Sensitivity is measured in scales established by official standards institutes following certain calibration protocols. The most popular used to be ASA, from the American Standard Association (at present, ANSI), and DIN, from its German counterpart. Currently both have been grouped together in the scale ISO: a film rated ISO $100 / 21^{\circ}$ is an ASA 100, DIN 21 film. It is important to note that ASA scale is linear, meaning that an ASA 200 film is twice as sensitive as an ASA 100 film. The DIN scale, on the contrary, is logarithmic: DIN degrees are ten times the decimal logarithm of the sensitivity, quite similar to decibel scale. Therefore, a film being twice as sensitive as another has a DIN value 3 units higher (as $\log 2 \cong 0.3$ ). DIN 21 is equivalent to ASA 100 , hence ISO $100 / 21^{\circ}$; ASA 200 would be DIN 24, hence ISO $200 / 24^{\circ}$ and so on. Often only one of the ISO numbers is given (ISO 200, ISO $24^{\circ}$ ); the degree symbol is included for former DIN scale to avoid confusions.

\section{2.c. Sensitivity in digital cameras}

The sensitivity concept can be expanded from film to digital cameras. The ISO range of analog cameras is between 200 and 1600; digital cameras usually feature a "native" sensitivity between 50 and 200 ASA, although sensitivities as high as 3 million can be found as well. Low ISO values require more light for a good exposure. If light level is not high enough, and the shutter speed cannot be reduced, then ISO must be raised. ISO can be increased by increasing the electric amplification of the signal collected by the sensor. This "trick" allows realizing photographs with low ambient light; however, noise increases as well, as it happened in film cameras with grain size. This noise is mainly perceivable in the darkest areas of the picture, where the amplification of the sensor noise becomes significant compared to the amplified signal. Moreover, if sensitivity is electronically increased, the dynamic range of the photograph grayscale is reduced. Therefore, the ability for collecting light in two sensors can be compared by looking at the minimum sensitivity specified by the manufacturer. Anyhow, a good SLR nowadays renders good quality pictures at ISO 1600 and above.

\section{2.d. Sensor size in digital cameras}

The amount of digital noise introduced at a given ISO level is affected by several factors, the sensor's pixel size being one of the most relevant: the larger the pixels, the lower the noise. The pixel size depends on the size and resolution of the sensor. At present, sensors of digital cameras are customarily offered in three formats: full frame $(36 \times 24 \mathrm{~mm})$, APS-C $(23.7 \times 15.6 \mathrm{~mm})$, and 2/3" CCD $(8.8 \times 6.6 \mathrm{~mm})$; The actual sizes may vary slightly depending on the sensor manufacturer. Sensors of smartphone cameras are smaller, currently between $1 / 3^{\prime \prime}$ and $1 / 2.3$ " in premium models (Fig. 2). This is the most obvious advantage of SLRs over compact and smartphone cameras, and the reason why SLRs perform better at higher ISOs.

When taking a "snapshot", the sensitivity is typically brought to the minimum value, and the other two parameters -shutter speed and f-number-are adjusted. The speed must be high to "grab" images of moving subjects; it may be low for static images or slow movements. Quasi-static images such as portraits or group pictures employ speeds of $1 / 60 \mathrm{~s}$ or higher. All in all, there is always a trade-off between noise, resolution, shutter speed and aperture. Recently, an Auto ISO feature has been included in several models, allowing the camera working in Auto mode to optimize not only the shutter speed and the aperture, but also change the ISO if required for obtaining a good shot. 
$35 \mathrm{~mm}$ "full frame"

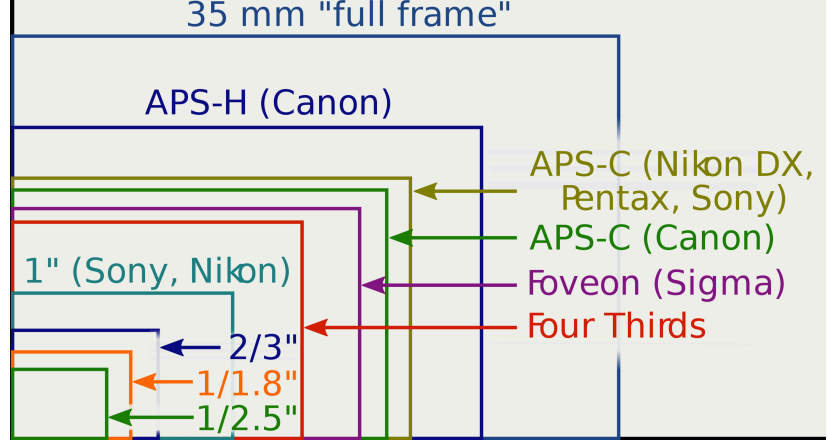

Figure 2. Relative size of common formats in digital cameras

\section{2.e. $f$-number}

The $\boldsymbol{f}$-number or $\boldsymbol{f}$ \# measures the amount of light collected by the objective per unit time when the shutter is open. It could be thought that this amount only depends on the aperture: the diaphragm aperture, obviously, determines the amount of collected light by enlarging or reducing the available lens area. However, the amount of light actually depends on the focal distance as well: the longer the distance, the lower the subtended angle from which light is captured; therefore, the objective is less luminous. The $\mathrm{f \#}$ is a ratio of these two parameters, the focal length and the diaphragm aperture. Mind that the ratio is inverse: the larger the $\mathrm{f} \#$, the smaller the aperture.

The $\mathrm{f \#}$ has a second effect on the picture: it determines the range of distances -behind and in front of the chosen focal distance- that appears focused as well. This range of distances is known as depth of field. As a consequence, for any given sensitivity, many pairs f\# - shutter speed can be used, resulting in pictures having the same luminosity but quite different compositions.

\section{The eye and the camera}

\section{3.a. Eye sensitivity}

Shutter speed and f\# are meaningless in the context of human vision (closing your eyes does not work as a shutter!). The eye has several mechanisms for regulating the amount of light and its effect on retina. Evolution has optimized these mechanisms over many millions of years, since having the best sight in extremely different scene lighting levels is often paramount for both, predators and preys, to survive.

The most obvious mechanism is widening and shrinking the pupils (similar to camera aperture); however, contrarily to popular belief, this is not the fundamental, but a mere accessory, short-term regulation mechanism. The pupil scarcely contributes to brightness regulation: indeed, the diameter of the pupil may vary, at most, from $8 \mathrm{~mm}$ in dark scenes to $1 \mathrm{~mm}$ in bright scenes. This is just a factor 64 in terms of area,

$$
\frac{\pi R^{2}}{\pi r^{2}}=\frac{4^{2}}{(1 / 2)^{2}}=64
$$

which is equivalent to a factor 64 , or $18 \mathrm{~dB}$, in luminance. The eye possesses simple yet powerful control mechanisms that allow humans to see in an amazing dynamic range of $10^{11}$ luminance levels $(110 \mathrm{~dB})$, adapting its photosensitivity to scenes from $10^{-6}$ lux (starlight in a moonless clear sky) to $10^{5}$ lux (bright sunshine in snow or beach). The ISO equivalent has been estimated in ISO 800 - ISO 1 [3].

The retina contains two kinds of photosensitive cells, rods and cones. There is a single type of rod and three types of cones; these are sensitive to different regions of the visible spectrum -consequently called S, M, L for short, medium, long wavelength- that allows photopic vision, in color. Rods provide scotopic vision, in black and white. Regarding sensitivity, rods are some 5-6 orders of magnitude more sensitive than cones, thus providing night vision, where cones cannot work, while rods are mostly saturated on daylight, where cones work. Below 3 lux only rods are active (scotopic vision); above 850 lux, only cones are active (photopic vision, rods are saturated). In the intermediate region, 3-850 lux (mesopic vision), both rods and cones are active. A side effect of this mechanism is that no colors are perceived below 3 lux, since rods are 
blind to colors. Cameras, on the contrary, are able to perceive color at any luminance (though their dynamic range is much narrower).

Besides this double mechanism, the range is further widened by a simple photobleaching strategy. Light is detected in retina by proteins, opsins, that contain a photosensitive molecule, retinal. Cone opsins are less sensitive than rod opsin, called rhodopsin or visual purple. When an opsin absorbs a photon, it delivers an electric signal through a photochemical cycle, and bleaches. This is the origin of bedazzlement when moving from a dark to a bright scene -and the temporal blindness in the opposite case. There is a reconstruction mechanism to regenerate the opsin; this process takes a certain time. As a consequence, the higher the luminance, the lower the amount of available, unbleached, opsins. Simple and efficient. There are cameras reaching these limits, but not this quasi dynamic range.

The pupil is useful for fast corrections of small luminance fluctuations; however, it does not have the range to compensate when abruptly getting out from a dark room to a sunny scene: you are dazzled until the pigment concentrations readjust to the correct levels.

There is another significant difference between eye and camera sensitivities: eyes work as a video recorder, getting many images over time. While eyes are moving about the scene, the brain extracts information for several different images, and the integration time varies with brightnes: it is low in photopic conditions, but reaches $10-15 \mathrm{~s}$ in darkest scenes. Cameras may accumulate photons for an unlimited time, allowing the sensor to render good pictures out of extremely dim scenes.

\section{3.b. Grayscale and color}

The human eye is overwhelmingly superior to cameras in dynamic range of illumination; however, this is not the case when considering gray levels. The number of gray levels perceived by a (trained) eye (e.g., a radiologist), may reach 150-200. A standard photographic film has analog grayscale, with theoretically infinity gray levels. A digital camera working in Raw format (without compression) may reach 14 bits (16384 levels), well over the human eye limit. Note: In order to benefit of the whole dynamic range of the film, pictures in classic photography used to be underexposed; this was eventually compensated in developing. However, in digital photography working in Raw format, it is advisable to slightly overexpose the Raw picture, eventually processing the file. This reduces the sensor thermal noise.

The eye sensitivity also varies with wavelength within the visible spectrum. The maximum sensitivity in photopic vision (cones) is centered in the green, at $555 \mathrm{~nm}$-an outcome of natural evolution in a planet whose solar spectrum is maximum precisely at that wavelength. However, the maximum sensitivity in scotopic vision (rods) is centered in the bluish-green, about $500 \mathrm{~nm}$. As a consequence, in mesopic vision the sensitivity performs a hypsochromic shift (towards shorter wavelengths). This is the reason why blue objects seem brighter than red objects in twilight like sunset (Purkinje phenomenon).

Finally, it is worth mentioning that eye vision is substantially free from many geometrical aberrations, but it does show chromatic aberration. It is not possible for the eye to focus all the colors onto the retina at the same time, since the refractive index of the visual system components is dependent on wavelength. When looking at a multicolor scene, green is customarily focused on the retina, while blue is focused at shorter distance, inside the eye, and red is focused behind the retina. Camera lenses are made as compensating doublets or triplets (achromatic lenses) to avoid this inconvenience.

\section{3.c. Projection of images in sensors and retina}

There are more remarkable differences between the optics of a camera and an eye. The image of the eye is projected onto a spherical surface. This is advantageous over the camera image, for it avoids some geometrical aberrations -e.g., the field curvature- and, at the same time, allows a uniform luminance on the retina (Fig. 1).

(Incidentally, the vertical eye section shown in Figure 1, like most eye sections shown everywhere, is wrong: the optic nerve in a vertical section is nearly centered, not under the eye axis as shown. What is sketched in the figure is a horizontal section of the left eye, seen from above. The optic nerve is nearly centered vertically but displaced towards the nose in both eyes.)

For an object to be focused onto the camera sensor, the lens must be moved forward and backward from the sensor (image plane). In the eye, focusing is achieved modifying the lens shape by changing its curvature. This focusing strategy is called accommodation (Fig. 3). 

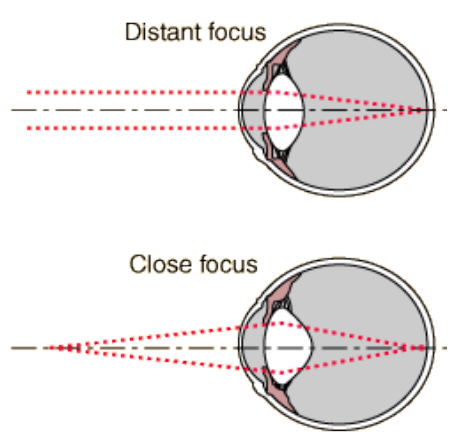

Figure 3. Accommodation and focusing
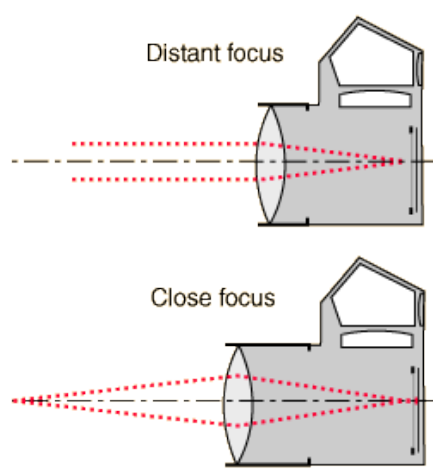

Three caveats must be announced here. First, for the sake of simplicity, we will refer to "a lens" within the course; a camera customarily employs a quite involved set of lenses. We will also use the approximation of thin lens. The same approximation in the eye is called the reduced eye of Listing (see below). Second, we are using lux as luminance units; this is not strictly correct, it should be called illuminance. Luminance is measured in $\mathrm{cd} / \mathrm{m}^{2}$ or nit, while lux $=$ lumen $/ \mathrm{m}^{2}=\mathrm{cd} \cdot \mathrm{sr} / \mathrm{m}^{2}$. This misuse is quite common and does not affect the contents of this course. Third, the concept of focal plane in photography differs from physics, and this may be confusing [5]. In physics (in Optics), the focal plane in thin lens approach is a plane perpendicular to the system axis located at the focal distance from the lens. The plane where image is formed is called the image plane. In photography, focal plane and image plane are often employed indistinctly. Actually, the image is formed at the physical focal plane only when the object is very far (infinity). However, the focal plane/image plane is defined as the plane where a formed image of an object is focused, i.e., sharp, and the position where the sensor is placed. We will use the term image plane where these different concepts may lead to confusions.

Both focusing methods are ruled by the so-called Descartes Law or Law of Conjugated Planes,

$$
\frac{1}{P}+\frac{1}{P^{\prime}}=\frac{1}{f}
$$

where:

- $\quad P \cong$ Distance object-lens

- $\quad P^{\prime} \cong$ Distance image-lens (sensor or retina, i.e., distance focal plane-lens in photographic argot)

- $f \cong$ Lens focal distance

The ratio $1 / f$ (where $f$ is given in meters) is known as lens convergence, and measured in diopters. The number of diopters of a lens is simply the inverse of the lens focal distance in meters.

When the distance to the object is varied, the eye varies $\mathrm{f}$ while the camera varies $\mathrm{P}^{\prime}$. If the camera features a zoom, $f$ will vary as well; nonetheless, this is done to modify the magnification, not the focusing, but usually re-focusing will be required as well. The longer the focal distance, the smaller the subtended angle, hence the larger the magnification. Focusing in a camera is always achieved by modifying the distance between the focal plane (sensor) and the lens.

Let us show the above ideas with some examples.

\section{3.c.1. Photographic camera}

Lens focal distance $=50 \mathrm{~mm}$.

When the lens focuses an object at $\infty$ (i.e., an object located very far), then:

$$
\frac{1}{\infty}+\frac{1}{P^{\prime}}=\frac{1}{f} \Rightarrow \frac{1}{P^{\prime}}=\frac{1}{f}
$$


Therefore, the focal distance of the lens coincides with the distance between the lens and the focal plane. If the object gets closer, the distance between the lens and the image plane must be increased for the equation to hold.

\section{3.c.2. Listing reduced eye}

The reduced eye of Listing is a simplification of the human eye (Fig. 4). The eye optics (cornea, lens, aqueous and vitreous humor) are described as an optical system having a thin lens with a distance of $17 \mathrm{~mm}$ to the focal plane.

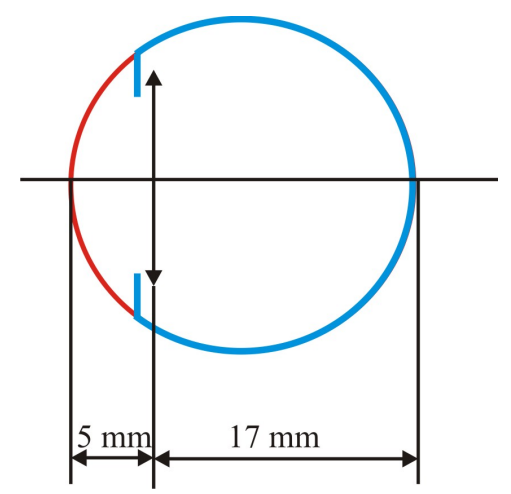

Figure 4. Listing reduced eye

It is customary to define, for a normal standard eye:

- Maximum focusing distance $\Delta=\infty \cong$ Punctum remotum and

- Minimum focusing distance $\delta=25 \mathrm{~cm} \cong$ Punctum proximum

Convergence for these two extreme cases is:

$$
\begin{gathered}
\Delta \Rightarrow \frac{1}{\infty}+\frac{1}{0.017}=\frac{1}{f}=58.8 \text { diopters } \\
\delta \Rightarrow \frac{1}{0.25}+\frac{1}{0.017}=\frac{1}{f}=62.8 \text { diopters }
\end{gathered}
$$

Considering the full convergence of the eye, and how it is achieved, one realizes that most diopters are provided by the cornea. The lens is responsible for 19 diopters, having a range of 4 diopters for focusing.

A shortsighted person has excessive eye convergence, making far images to focus in front of the retina when focusing to infinity. If that person wears correction glasses (divergence lenses) of, say, 2.5 diopters, the minimum convergence of his/her eyes shall be:

$$
1 / f_{\min }=58.8+2,5=61.3 \text { diopters }
$$

And the maximum distance the person is able to focus without glasses is:

$$
\Delta \Rightarrow \frac{1}{X}+\frac{1}{0.017}=61.38 \text { diopters } \Rightarrow X=40 \mathrm{~cm}
$$

The above explanation notwithstanding, there is a certain distance threshold for which the brain accepts the image as focused, even being not completely sharp. This "acceptable defocusing" is known as depth of field (DoF). This parameter depends on several factors, as seen below.

\section{Visual acuity and eye resolution}

\section{4.a. The eye visual acuity}

Visual acuity is the ability of the eye to distinguish shapes and details of objects at a given distance. In other words, visual acuity is a measurement of the spatial resolution of the visual processing system. It can be measured using optotypes -sets of letters, symbols or figures- as employed by opticians (giving results like $20 / 20$ or $6 / 6$ ). Acuity in optics is given as a single number, the inverse of an angular measurement in arcminutes, referred to the projection of the scene onto the retina. Some different patterns have been proposed 
for this measurement leading to different results; at present, the most popular pattern is a set of equidistant lines, whose thickness equals their gaps, and the measurement refers to a line pair (LP, a black line and a white gap) subtending an angular span -called the visual angle or the apparent diameter, see belowrequired for the eye to distinguish between a black/white pattern and a plain continuous gray. LPs lead to the most important issue for comparing eyes and cameras, the concept of spatial frequency shown below.

The visual acuity is extremely dependent on lighting -this is why we use a bright light to perform any action on tiny objects like needles. The highest acuity is reached for luminance above 3000 lux, well in the photopic range. This maximum acuity is only reached at the fovea (Fig. 5), precisely the retina region populated only by cones. The accepted angular values are in the range of 0.2-0.3 mrad, i.e., 0.6 to 1.0 arc-minutes. This is equivalent to an acuity of 1-1.7. The section of cones in fovea is roughly $2.5 \mu \mathrm{m}$. A pair of cones, therefore, has about $5 \mu \mathrm{m}$. The visual acuity corresponds to the angular separation of these two cones. In other words, the eye (actually the fovea under high lighting levels) is able to discriminate a broken line of black dots and white gaps just having the same angular span as the cone separation.
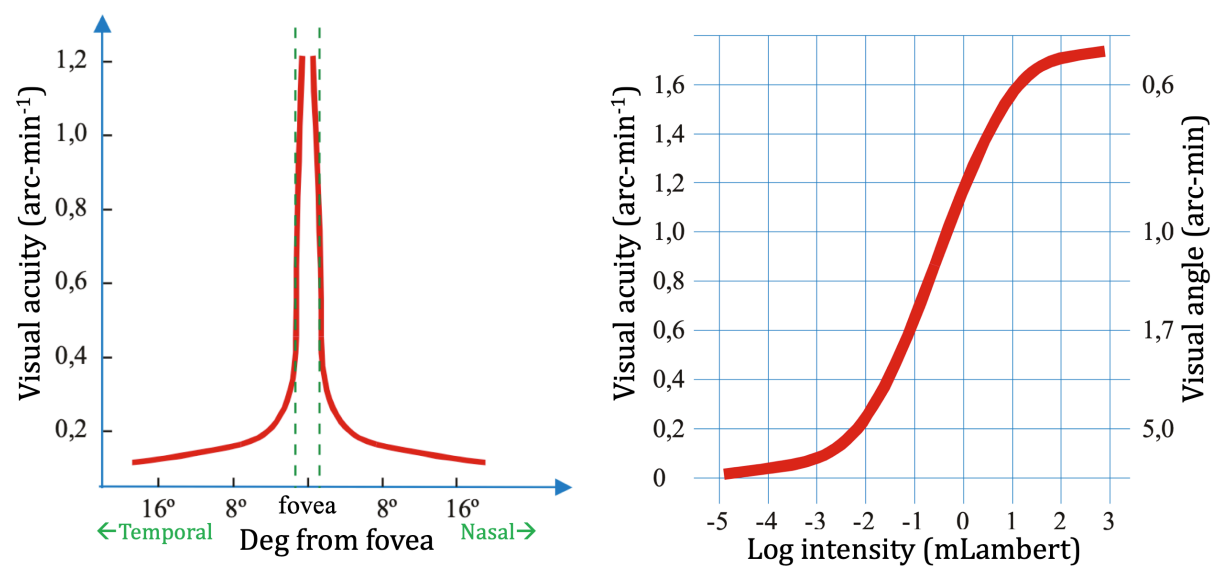

Figure 5. Visual acuity. Left, as a function of angular distance from fovea. Right, as a function of light intensity. 1 millilambert $=10 / \pi \mathrm{cd} / \mathrm{m}^{2}$

\section{4.b. Eye resolution: apparent diameter}

Eye resolution is closely linked to acuity. A classic example known for centuries is the second star of the handle of Ursa Maior constellation, which is actually a binary system (Mizar and Alcor). People able to distinguish both stars have been traditionally considered to possess excellent eye resolution.

The eye resolving power depends on several factors:

- The person

- The lighting

- Fatigue

- Object contrast

The eye resolving power is customarily measured through the apparent diameter; as pointed above, this is actually an angular measurement rather than a length. The apparent diameter (Fig. 6) is the minimum observation angle, $\Sigma$, in radians, required for the eye to separate two points. Note: apparent diameter is also known as angular diameter, angular size, apparent size, visual angle (in vision) or angular aperture (in Optics).

$$
\Sigma(\mathrm{rad})=\theta=\arctan \frac{e}{d}
$$


Being a minimum observation angle, the angle $\Sigma$ is quite small. This allows equaling the angle in radians and the tangent; therefore:

$$
\Sigma(\operatorname{rad}) \approx \frac{e}{d}
$$

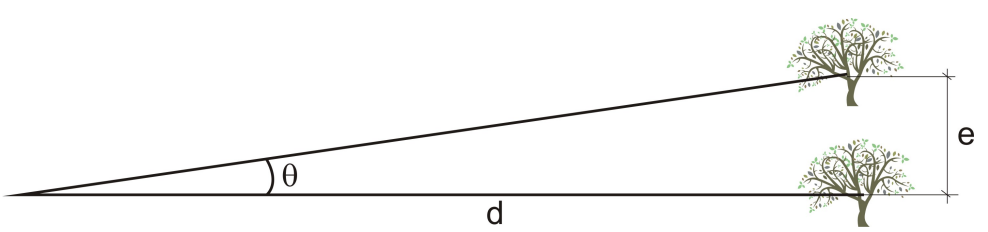

Figure 6. Apparent diameter

" $e$ " introduces the idea of spatial frequency. Looking at a line chart, with equally spaced fringes, at increasing distance, there will be a distance " $d$ " above which the chart looks like containing a homogeneous gray. The Nyquist criterion establishes that lines separated " $2 e$ " are perceived at a maximum distance " $d$ ".

\section{Resolution of a camera}

\section{5.a. Line chart measuring}

The concept of camera resolution is similar to eye resolution. The resolution of a camera can be measured using a line chart as well (Fig. 7). It depends on:

- Objective aberrations

- Diaphragm aperture

- Film grain or sensor resolution

The separation between fringes in a line chart is given as:

- Line pairs per $\mathrm{mm}(\mathrm{LP} / \mathrm{mm})$ in space

- Number of cycles per $\mathrm{mm}(\mathrm{CL} / \mathrm{mm})$ or $(\mathrm{Hz} / \mathrm{mm})$ in frequency. This is often used when employing sinusoidal patterns rather than white and black fringes.

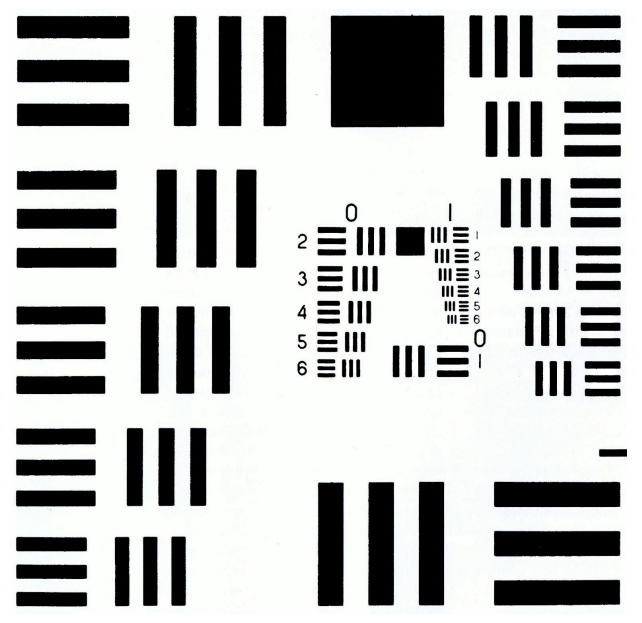

Figure 7. Line chart USAF 1951

Measuring the resolution of a film camera is simple: take a picture of the chart and looking at the negative in a microscope. With an appropriately chosen film, the limiting factor will always be the camera, and not the image recording media.

The resolution of a digital camera image is assessed using sufficiently enlarged pictures.

Measuring the resolution of a digital camera is more involved, since the resolution is usually worse than one could expect without further ado. The problem derives from the regular distribution of the pixels, which may or may not coincide with the projection of the fringes. 


\section{5.b. Perceived sharpness of the final image: the Modulation Transfer Function}

The quality perception of an image is affected by the perceived sharpness, or apparent sharpness, which in turn depends on:

- The optical resolution

- The Modulation Transfer Function (MTF)

- The subjective psychological response

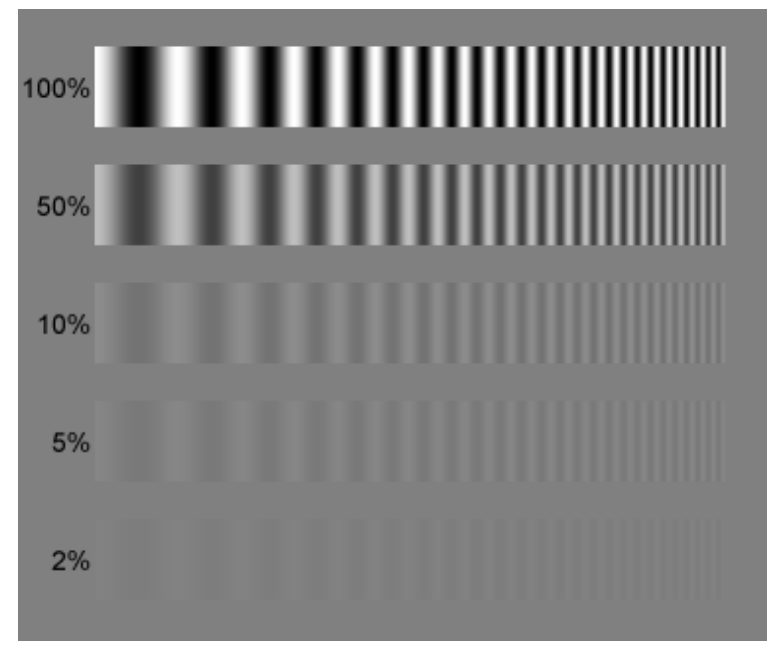

Figure 8. Sinusoidal signal for different spatial frequencies and contrast values (www.normankoren.com)

Image sharpness depends on resolution, and also on contrast. As a rule, taking two pictures with the same resolution, sharpness is higher for the picture having higher contrast.

The Modulation Transfer Function or MTF parameter (also known as spatial frequency response) was introduced to specify image sharpness in an objective way. MTF is the contrast (normalized to low spatial frequency contrast) as a function of the spatial frequency. Its value is 1 at low frequency and lower at higher frequency. From other point of view, MTF can be defined as the response to a step function; in this case is known as Contrast Transfer Function (CTF).

Test charts for MTF calculation in photographic systems are usually made of sinusoidal patterns (Fig. 8) where the spatial frequency is continuously varied. MTF (Fig. 9) is obtained from the Fourier transform of the image. If a line chart is used, the CTF is obtained.

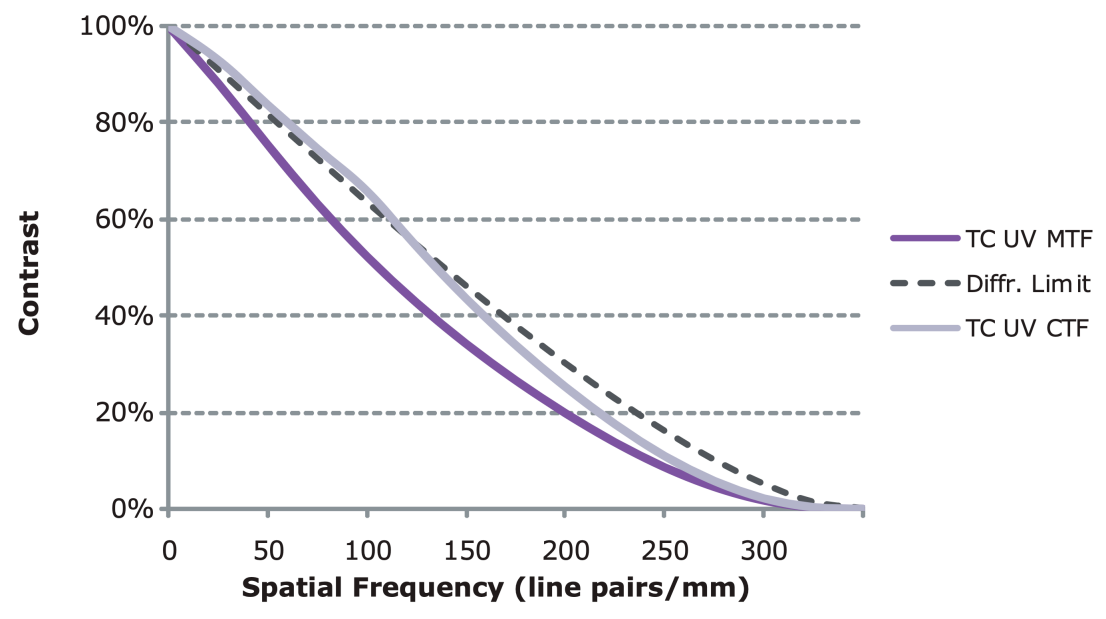

Figure 9. MTF measured in the center of a lens (TC-UV, http://www.telecentric-company.com) vs. spatial frequency (LP/mm). The gray curve is the response to a line chart $(\mathrm{CTF})$, the purple curve is the response to a test chart of sinusoidal patterns (MTF), and the dashed line is the diffraction limit. 


\section{5.c. MTF and analysis of spatial frequencies}

The MTF of a camera depends on the position on sensor, or film. The quality of the image will degrade as one moves from the center of the photograph to the corner. Most manufactures of camera objectives provide data about the MTF values as a function of the distance from the center.

Apart from spatial frequency, the MTF is intrinsically a function of illumination, of aperture and f\#. The manufacturer will typically declare the relevant conditions, but will tend to have chosen optimum conditions for the characterization of a specific lens.

At least two spatial frequencies are chosen (typically a low frequency of $10 \mathrm{LP} / \mathrm{mm}$ and a higher frequency of $30 \mathrm{LP} / \mathrm{mm}$ ). For each frequency the variation of MTF is shown as a function of diagonal distance from the center reaching the corner of the sensor (or photograph) (Figure 10). Typically the sensor is tested with a test pattern in which the lines run parallel the line running from the center of the image to the corner (sagittal), and on lines are perpendicular to that diagonal (meridional) [6].

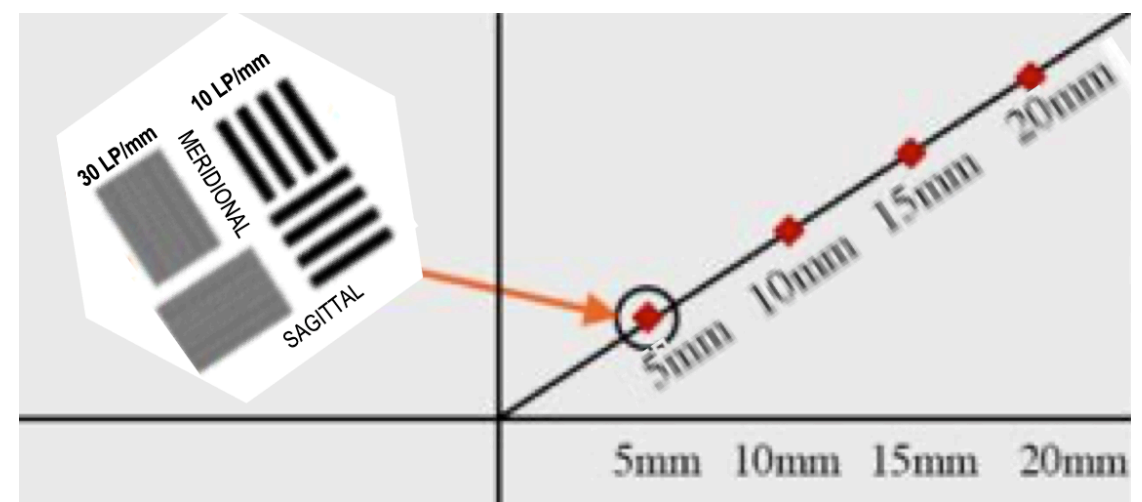

Figure 10. MTF of a lens (TC-UV, http://www.telecentric-company.com) measured at different distances of the diagonal using Meridional (perpendicular) and Sagittal (parallel) patterns with respect to the diagonal direction. The patterns are low $(10 \mathrm{LP} / \mathrm{mm})$ and higher $(30 \mathrm{LP} / \mathrm{mm})$ spatial frequencies.

Graphs are customarily shown with thick lines for low frequency $(10 \mathrm{LP} / \mathrm{mm})$ and thin lines for high frequency $(30 \mathrm{LP} / \mathrm{mm})$. Alternatively different colors are used. In Figure 11, $10 \mathrm{LP} / \mathrm{mm}$ is shown in red and $30 \mathrm{LP} / \mathrm{mm}$ is shown in blue.

The above MTF analysis is actually presenting three parameters of the objective: the contrast with the 10 $\mathrm{LP} / \mathrm{mm}$ line, the resolution with the $30 \mathrm{LP} / \mathrm{mm}$ line, and the astigmatism with the difference between meridional and sagittal lines.

As mentioned MTF analysis depends on the diaphragm. Hence, the analysis is performed at fixed apertures, typically the maximum aperture supported by the cell yet sometimes at $\mathrm{f} / 8$ (it is considered that $\mathrm{f} / 8$ corresponds to the diaphragm aperture that gives better sharpness. At lower $\mathrm{f}$ the aberrations increase and at higher $\mathrm{f} \#$ the effect of diffraction reduces the sharpness). The results depend on the focal distance as well. For this reason, in the more complete characterizations MTF is measured for zooms corresponding to both the extreme focal distances.

The ideal resultant MTF would be 1 from the center to the corner. In practice, an objective whose contrast (10 LP/mm resolution) is above 0.8 over the whole diagonal may be considered excellent; over 0.6 is acceptable. On the other hand, if the solid and the dashed lines are very dissimilar, then the meridional and sagittal behaviors are different; this means that the objective has a noticeable astigmatism. This becomes apparent when defocusing generates defocused areas with concentric lines. A circular diaphragm and a low astigmatism generate images with better bokeh (defocusing quality). A good bokeh makes the defocused areas of the picture more pleasant.

Coming back to Figure 11, the MTF of the Nikkor 105/2.8 VR shows contrast lines (10 LP/mm, red) above 0.9 all over the photogram. Besides this, the solid and dashed red lines match for virtually the full diagonal, apart from the extreme corners. These features demonstrate that the objective is excellent and should give a good bokeh -providing the diaphragm is circular enough. The blue lines $(30 \mathrm{LP} / \mathrm{mm})$, are fairly horizontal above 0.6, meaning a good resolution if the camera employs APS-C format. The resolution is compromised in the corners if the camera uses full-frame format $(35 \mathrm{~mm})$. 


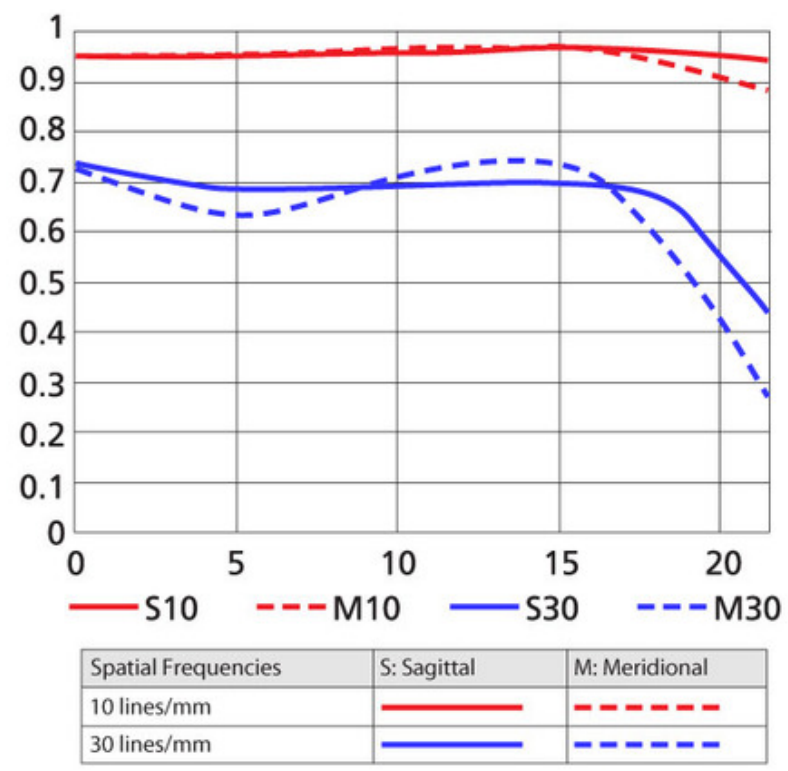

Figure 11. MTF graph of the Nikkor 105/2.8 VR, Solid lines refer to sagittal orientations and dashed lines to meridional orientations.

\section{5.d. System and perception MTF}

How can the MTF be related to the resolution in $\mathrm{LP} / \mathrm{mm}$ ? The resolution in $\mathrm{LP} / \mathrm{mm}$ corresponds to an MTF of about 2\% - 5\% (0.02-0.05); this value may vary between different observers. The Rayleigh diffraction criterion, mentioned below, is linked to an MTF of 9\%.

Each element of a camera has its own MTF. The overall MTF is calculated multiplying the MTFs of each element. This can be applied to other optic systems such as displays, printers, and scanners; the eye has its own MTF as well. It is interesting to mention that eye contrast sensitivity varies with spatial frequency: at low and high frequencies, the human eye needs more contrast to appreciate the light-dark oscillations. The maximum visual MTF (i.e., the least required contrast) is about 5 cycles/ ${ }^{\circ}$ (Fig. 12). This variation is responsible for many edge enhancements of human vision and, collaterally, for many optical illusions.

Besides contrast and resolution, there is another parameter that remarkably affects the sharpness perception: acutance. Acutance is the edge contrast of an image; hence it accounts for the changes (derivative) of brightness within the picture. Abrupt changes imply high acutance. Sharpness can be

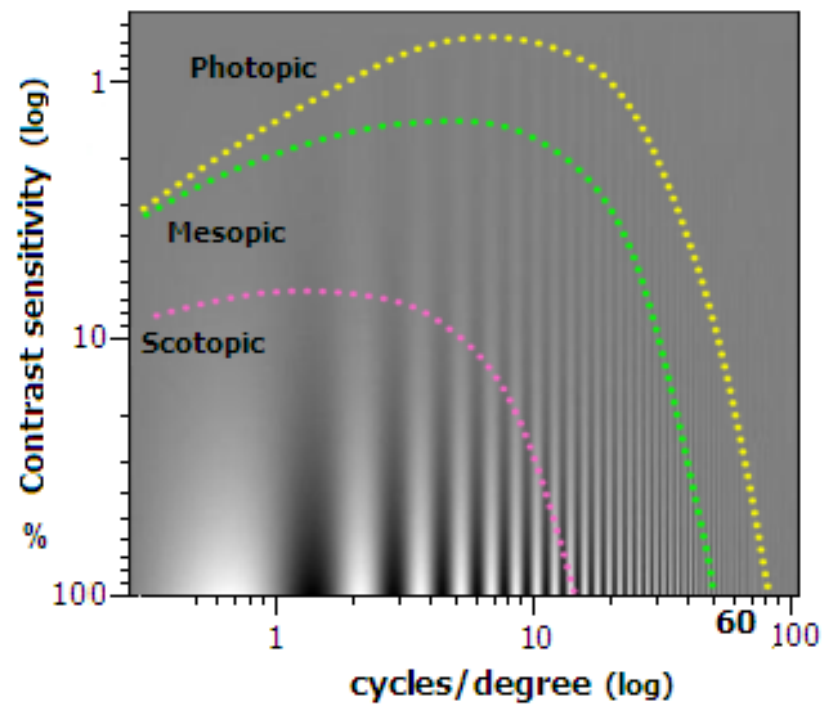

Figure 12. Human Contrast Sensitivity Function, a.k.a. visual system MTF. Above each curve, bands are undistinguishable, below are distinguishable; note the log-log scale. 
increased without increasing the resolution, by enhancing the edges of the picture objects with darker lines in dark areas adjacent to brighter lines in bright areas. This is a technique well known by painters (and a mechanism habitually applied by the eye). The regular distribution of pixels in digital cameras (also in displays) may originate "oversharpness" of the pictures, somewhat losing their natural view.

\section{Image resolution: some examples}

Let us calculate the resolution required for a sensor to blow up an image to the maximum resolution distinguished by the eye. We will apply the calculation to three sensors: Full Frame $(24 \times 36 \mathrm{~mm})$, APS-C $(23.7 \times 15.6 \mathrm{~mm})$ and $2 / 3^{\prime \prime} \mathrm{CCD}(8.8 \times 6.6 \mathrm{~mm})$.

\section{6.a. Eye resolving power}

The minimum focal distance for a normal eye is $25 \mathrm{~cm}$. Considering a visual acuity of 1 , the resolving power is 1 arc-minute or $0,3 \mathrm{mrad}$. Therefore:

$$
0.0003 \mathrm{rad}=\frac{e}{250 \mathrm{~mm}} \Rightarrow e=0.0003 \cdot 250=0.075 \mathrm{~mm}
$$

The eye will be able to see two distinct lines rather than a gray blur when the distance between lines is at least:

$$
0.075 \cdot 2=0,15 \mathrm{~mm}
$$

Consequently the maximum eye resolution -assuming an observation distance of $25 \mathrm{~cm}$ - is:

$$
\frac{1}{0.15 \mathrm{~mm}}=6.67 \mathrm{LP} / \mathrm{mm}=169 \mathrm{LPI}(\text { Line Pairs } / \text { inch })
$$

Note: Do not confuse the LPI and the printer resolution given in DPI (dots per inch). For a printer to define a line, it requires at least 7-8 dots, i.e., 16 dots per line pair (Fig. 13). This gives a rough estimation of the minimum resolution.

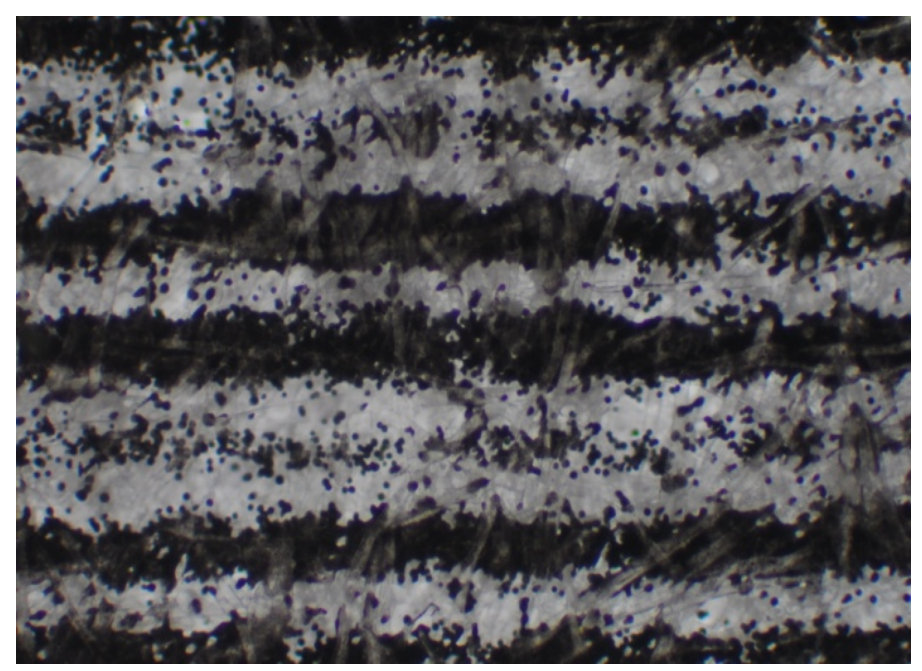

Figure 13. Microscopic view of a line chart with 50 LPI as printed by a laser printer. Note that the printer is not able to define all lines with this resolution. Also note that (the thickness of) each line is defined by 8-10 dots.

\section{6.b. Format and enlargement factor}

Let us assume that we want to enlarge a picture to fit to a DIN A4 sheet, i.e. $300 \times 210 \mathrm{~mm}$.

We will perform the same calculation for the three sensor formats mentioned above: full frame $(36 \times 24$ $\mathrm{mm})$ employed by high-end reflex cameras; APS-C $(23.7 \times 15.6 \mathrm{~mm})$ employed by standard reflex cameras, and $2 / 3^{\prime \prime}$ CCD $(8.8 \times 6.6 \mathrm{~mm})$ used in compact digital cameras. The APS-C size corresponds to a sensor DX 
of Nikon, or sensors from Pentax and Sony. Canon utilizes a slightly smaller sensor. Olympus, Panasonic, Kodak, and Leica use even smaller sensors, with 4/3 format (see Figure 2).

Choosing the longer side, the enlarging factor is:

$$
\begin{aligned}
& \text { Full Frame }(24 \times 36 \mathrm{~mm}) \Rightarrow 300 / 36=8.33 \text { times } \\
& \begin{array}{l}
\text { APS-C }(23.7 \times 15.6 \mathrm{~mm}) \Rightarrow 300 / 23.7=12.65 \text { times } \\
2 / 3 " C C D(8.8 \times 6.6 \mathrm{~mm}) \Rightarrow 300 / 8.8=34 \text { times }
\end{array}
\end{aligned}
$$

\section{6.c. Sensor resolution}

Table 1 summarizes the maximum pixel size that different sensors can have. Note that the calculation of maximum pixel size requires the number of line pairs per $\mathrm{mm}$ to be multiplied by 2 . This is because at least

\begin{tabular}{|c|c|c|}
\hline & \multirow[b]{2}{*}{$\mathrm{LP} / \mathrm{mm}$} & \multirow[b]{2}{*}{$\begin{array}{c}\text { Maximum } \\
\text { pixel size }(\mu \mathrm{m})\end{array}$} \\
\hline & & \\
\hline Full Frame $(24 \times 36 \mathrm{~mm})$ & $6.66 \mathrm{LP} / \mathrm{mm} \cdot 8.33=55.55$ & $1 /(2 \cdot 55.55)=9$ \\
\hline APS-C $(23.7 \times 15.6 \mathrm{~mm})$ & $6.66 \mathrm{LP} / \mathrm{mm} \cdot 12.65=84.33$ & $1 /(2 \cdot 84.33)=6$ \\
\hline $2 / 3^{\prime \prime} \mathrm{CCD}(8.8 \times 6.6 \mathrm{~mm})$ & $6.66 \mathrm{LP} / \mathrm{mm} \cdot 34=226$ & $1 /(2 \cdot 226)=2.2$ \\
\hline
\end{tabular}
two pixels are required to define a line and a gap between lines.

TABLE 1. Resolutions of several sensors

This calculation is reasonable for traditional film, but unfortunately it overestimates digital cameras. The reason is that digital devices have regularly distributed pixels. Unless lines and gaps coincide exactly with consecutive pixel lines, the lines will be blurred for they will affect contiguous pixels. Consequently the sensor resolution must be increased to achieve the same picture resolution. Obviously this problem only arises when working with a line chart or other regular pattern. The calculation above is roughly correct for a "normal" picture. In this case, resolution will be compromised just in areas having horizontal or vertical lines. The regular distribution of pixels is partially responsible for aliasing in digital cameras. (Some aliasing is due to the use of color filters as well.)

The number of pixels for each sensor can be derived from the results of the previous calculations. If the aspect ratio were the same, the resolution would obviously be the same for the three sensors, since it is defined by the pixels we have defined in the original A4.

$$
\begin{aligned}
& \text { Full Frame }(24 \times 36 \mathrm{~mm}) \Rightarrow(36 \cdot 24) / 0.009^{2}=10.7 \text { Mpixels } \\
& \text { APS-C }(23.7 \times 15.6 \mathrm{~mm}) \Rightarrow(23.7 \cdot 15.6) / 0.006^{2}=10.2 \text { Mpixels } \\
& 2 / 3^{\prime \prime} \mathrm{CCD}(8.8 \times 6.6 \mathrm{~mm}) \Rightarrow(8.8 \cdot 6.6) / 0.0022^{2}=12 \text { Mpixels }
\end{aligned}
$$

The image size is quite moderate and similar in all cases. Apparently, there is no reason for using expensive large sensors in high-end cameras. Actually, there are two reasons for preferring large sensors. First is sensitivity: the larger the sensor, the larger the amount of collected light. Second is diffraction effects, as seen in next section.

\section{Color resolution and color temperature}

Eye resolution for colors is much smaller than its resolution for brightness. In other words, the eye discriminate black and white lines or spots much more efficiently than color lines, i.e., the color visual acuity is worse than the $\mathrm{B} / \mathrm{W}$ acuity. Every active rod and cone play a role in brightness resolution; however, only the cones for the specific color participate in color resolution. As a result, eye resolution for color separation between primaries is significantly lower. Resolutions for a standard eye are shown in Table 2.

TABLE 2. Color resolutions of human eye

\begin{tabular}{|l|c|c|}
\cline { 2 - 3 } \multicolumn{1}{c|}{} & Resolution (arc-minutes) & Resolution (mrad) \\
\hline Luminance & 1.0 & 0.3 \\
& 4.1 & 1.2 \\
& 6.9 & 2.0 \\
\hline
\end{tabular}




\begin{tabular}{|l|c|c|}
\hline Between red and green & 8.6 & 2.5 \\
Between red and blue & & \\
Between green and blue & & \\
\hline
\end{tabular}

This is the reason why a camera does not need three color subpixels per pixel; color representation requires a lower color resolution. Mosaic filters (also known as Bayer filters) of three or four pixels are customarily employed.

The use of mosaic filters has a collateral problem: each filter only allows the transmission of its color; the camera must estimate the pixel brightness (the gray level the pixel would have without filter). This is done with interpolation algorithms taking into account the brightness of the neighboring pixels and the "weight" of each filter in overall luminance. Color is also calculated by interpolation of adjacent pixels; this may produce chromatic aliasing.

The final stored camera image is not the image captured by the sensor, but a processed image containing full and independent information of gray level and color of every pixel.

Light has a specific spectrum determined by its color temperature. For the eye, the brain automatically corrects colors depending on the light color temperature. This brain processing is the reason why one can correctly identify most colors while wearing, e.g., green sunglasses. An analog film camera needs external color filters; a digital camera utilizes an electronic correction (white balance). Notwithstanding all these correction mechanisms, scenes taken in fluorescent or LED lighting often look greenish or bluish, since fluorescent lights do not emit the whole continuous white spectrum, but rather a set of primary RGB color bands, the sum of which the human eye perceives as white (the same effect is used for generating colors in TV displays)

\section{Light diffraction}

\section{8.a. Field of View and f-number}

The amount of light impinging on the sensor depends chiefly of two factors: the focal length (or focal distance) and the diaphragm aperture.

The longer the focal length is, the smaller the light entrance angle. The gathered light is lower, and the image is larger. The entrance angle of light reaching the sensor is called Field of View (FoV) or field angle.

Figure 14 shows the effect of FoV on two lenses having different focal lengths and focused to infinity.
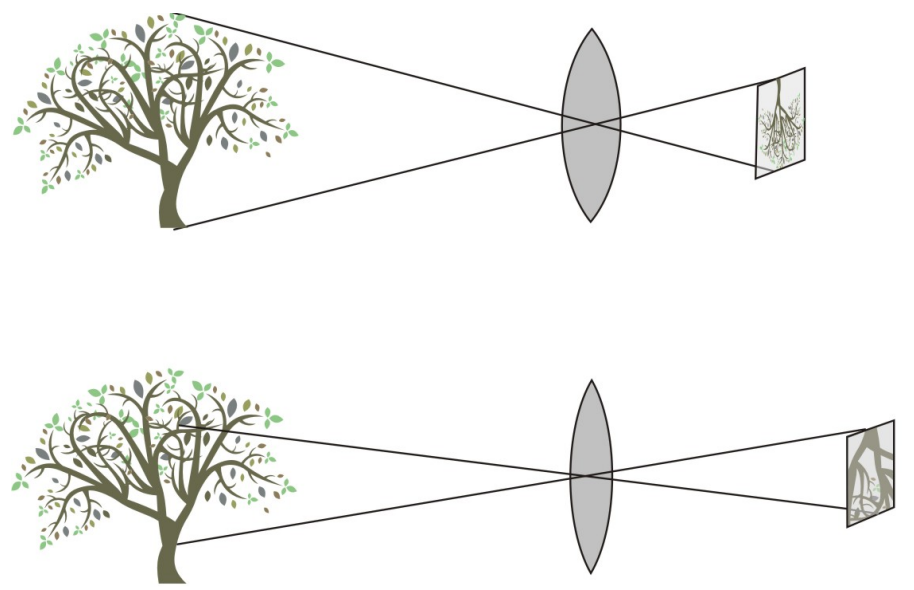

Figure 14. Effect of the focal distance on the encompassed Field of View

The amount of light reaching the sensor is determined with the $\mathrm{f}$-number or $\mathrm{f} \#$. As mentioned above, $\mathrm{f} \#$ is defined as the ratio $f / n$, where

$$
n=\frac{\text { Focal distance }}{\text { Diaphragm aperture diameter }}
$$


The ratio $f / n$ gives the diaphragm aperture diameter while $n$ indicates the luminosity of the objective for a given pair of distance and diaphragm aperture. However, in optics jargon, it is customary to employ the term $f$-number rather than $n$-number.

For example, a picture taken with $f / 2.8$ and a focal length of $50 \mathrm{~mm}$ would use a diaphragm diameter of: Some additional considerations must be taken into account:

$$
\phi=50 \mathrm{~mm} / 2.8=17,85 \mathrm{~mm}
$$

- The diaphragm aperture can be calculated, but is not relevant. What really matters is the amount of light passing the objective; this is given by f\#. For example, if we are using an objective with zoom -hence variable focal length- it is possible to obtain the same luminance for different combinations of focal lengths and diaphragm apertures.

- The collected light is lower as the $f \#$ increases. This is achieved either closing the diaphragm or increasing the focal length.

- The minimum $f \#$ of an objective gives an idea of its luminosity. Smaller $f \#$ 's indicate that the objective is more luminous (and much more expensive!)

- Cameras usually work in "f-stops" or "light steps" (or colloquially "stops"). Each step doubles the amount of light. The sensor or film sensitivity is given in ISO values: 50, 100, 200, 400, 800, etc., each value doubling the preceding one. Exposure times are given in fractions of second, 1/30, 1/60, $1 / 125,1 / 250,1 / 500$, etc., each value halving the preceding one.

- Just as a curiosity, the dynamic range of the human eye would be about $37 \mathrm{f}$-stops.

The same rule is applied to $f \#$. The amount of light is doubled when the diaphragm diameter is multiplied by $\sqrt{2}$. This is why $f \#$ 's are given in powers of $\sqrt{2}: f / 0,7, f / 1, f / 1,4, f / 2, f / 2,8, f / 4, f / 5,6, f / 8, f / 11, f / 16, f / 22$.

\section{8.b. Diffraction limit}

When an opaque object is placed between a light source and a screen, the projected shadow is not sharp: the shadow area always contains some light. The same happens when light passes through a slit. Without digging into many details, the phenomenon is called diffraction and can be explained in terms of the Huygens principle: the propagation of a wavefront can be predicted assuming that every point reached by the wave becomes a new wave source emitting in all directions. The sum of all new waves will be the new wavefront; it is calculated using the superposition principle.

A quantitative approach to diffraction is given by the Fresnel-Kirchhoff formula. The mathematical treatment is quite complex and certainly beyond the scope of this course. However, there are two approximations that may remarkably reduce the calculations:

- Fresnel or near-field diffraction. Only valid for very short distances, it assumes a curved wavefront

- Fraunhofer or far-field diffraction. Only valid for very long distances, it assumes a flat wavefront

There is not a clear separation between both approaches; anyhow, the selection becomes obvious in many actual situations. Let us show some examples that may result useful for evaluation of diffraction in the optical system of a camera.

\section{8.b.1. Diffraction in a slit.}

Light passing through a slit of width $D$ is projected onto a screen. Let us assume far-field, i.e., the distance between the slit and the screen is much longer than the slit width. Applying the superposition principle, a set of maxima and minima is obtained. The position of these maxima and minima is given by:

$$
\sin \theta=\frac{P \cdot \lambda}{D}
$$

where $P$ is the order of the maximum or minimum. Therefore, the position of the first minimum is

$$
\sin \theta=\frac{\lambda}{D}
$$

and the first maximum is at: 


$$
\sin \theta=\frac{2 \cdot \lambda}{D}
$$

The set is symmetric at both sides of the slit.

\section{8.b.2. Diffraction in a circular hole.}

The calculation for a circular hole is identical, with the only difference of having circular, rather than linear, symmetry. The diffraction of a circular hole is a pattern of alternating bright and dark circles known as Airy disk (Fig. 15).

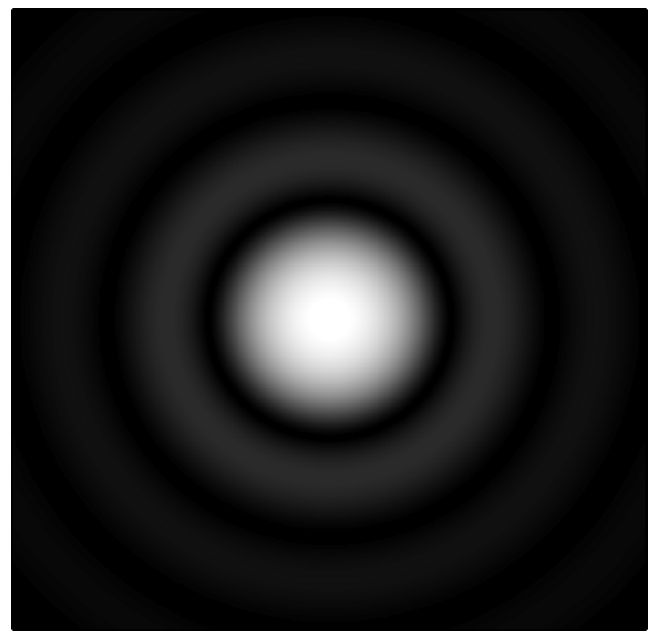

Figure 15. Calculated Airy disk

If the diameter of the hole is $D$, maxima and minima of the diffraction pattern will be located at some specific angles from the normal, given by:

$$
\sin \theta=1.22 \cdot \frac{P \cdot \lambda}{D}
$$

where $D$ is the diameter and $P$ is the order of the maximum or minimum. The diffraction image of a circular hole is known as Airy disk (Fig.15)
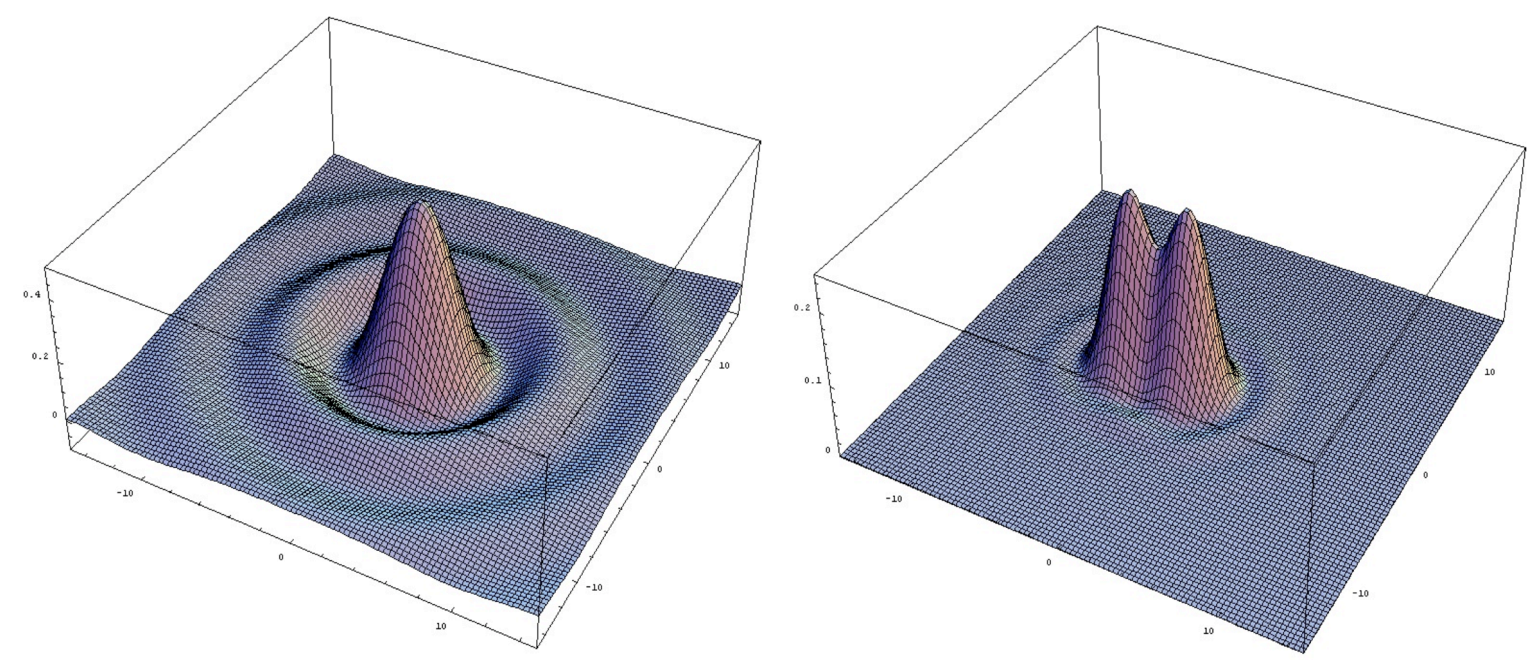

Figure 16. 3D Airy function (left) and Rayleigh criterion for distinguishable points (right) [7] 
If light is passing through two holes simultaneously, the holes will be distinguishable or not depending on their size and relative distance. The exact limit for two light sources (the holes) to be distinguishable or not is certainly subjective. Some objective criteria may be applied, though. The most popular is the Rayleigh criterion (Fig. 16). It sprouts from the human vision capability and was initially proposed to distinguish light points (stars) in a telescope. The Lord Rayleigh criterion is simply an empirical rule establishing that two point sources can be distinguished when the maximum of the first source matches the minimum of the second source and vice versa, i.e., that the distance between elements correspond to the first Airy minimum $(P=1)$.

This criterion is equivalent to a minimum contrast of $9 \%$ between sources.

Note that this or any other criterion effectively limits the minimum distance between two objects for them to be distinguishable at a given distance and a specific objective aperture. Moreover, in any sensor (not in the eye), the criterion determines the maximum resolution that can be reached under these circumstances, independently of the intrinsic resolution of the sensor (that may be higher).

$1^{\text {st }}$ Example: A telescope with a lens having $10 \mathrm{~cm}$ of diameter. What is the minimum separation between two distinguishable objects at $500 \mathrm{~m}$ ?

Let us assume a wavelength $\lambda=550 \mathrm{~nm}$, in the green region. Following the Rayleigh criterion (Fig. 17), and taking into account that sine, tangent, and angle can be interchanged for very small angles,

$$
\sin \theta \approx \tan \theta=\frac{e}{d} \approx 1.22 \cdot \frac{P \cdot \lambda}{D} \Rightarrow e=\frac{1.22 \cdot 1 \cdot \lambda \cdot d}{D}=\frac{1.22 \cdot 550 \cdot 10^{-9} \cdot 500}{0,1}=3.4 \mathrm{~mm}
$$

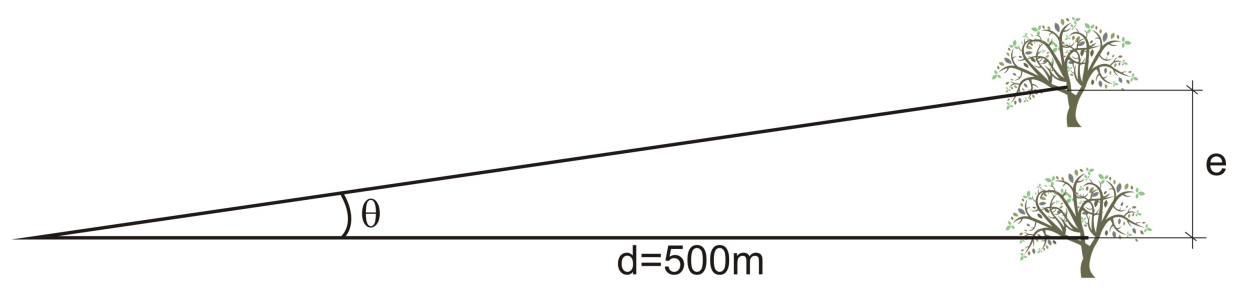

Figure 17. Rayleigh criterion for a telescope

$2^{\text {nd }}$ Example: Consider a camera focused to infinity. What is its maximum resolution as a function of the diaphragm aperture?

If the camera is focused to infinity, then $p^{\prime}=f$, i.e. the focal length of the lens coincides with the distance to the focal plane

$$
\sin \theta \approx \tan \theta=\frac{e^{\prime}}{p^{\prime}}=\frac{e^{\prime}}{f} \approx 1.22 \frac{P \cdot \lambda}{D} \Rightarrow e^{\prime}=\frac{1.22 \cdot 1 \cdot \lambda \cdot f}{D}=1.22 \cdot 550 \cdot 10^{-9} \frac{f}{D}=0.671 \cdot n[\mu \mathrm{m}]
$$

For example, closing the diaphragm at $\mathrm{f} / 8$, the minimum separation between two distinguishable points would be $0.671 \cdot 8=5.37 \mu \mathrm{m}$ and the maximum resolution would be

$$
1 / 5.37 \mu m=188 \mathrm{LP} / \mathrm{mm}
$$

However, closing the diaphragm to $\mathrm{f} / 22, e^{\prime}=15 \mu \mathrm{m}$, and the maximum resolution would be

$$
1 / 15 \mu \mathrm{m}=46 \mathrm{LP} / \mathrm{mm}
$$

Coming back to the three sensor formats previously mentioned, the pixel sizes as seen in Table 1 were:

- Full frame $(24 \times 36 \mathrm{~mm}) \Rightarrow 9 \mu \mathrm{m}$

- $\quad$ APS-C format $(23 . \times 15.6 \mathrm{~mm}) \Rightarrow 6 \mu \mathrm{m}$

- $2 / 3^{\prime \prime}$ format $(8.8 \times 6.6 \mathrm{~mm}) \Rightarrow 2.2 \mu \mathrm{m}$ 
None of the above sensors would reach the nominal resolution with a diaphragm $f / 22$. This is the reason why cameras employing low-size sensors -such as smartphones- cannot use high $f f^{\prime}$ 's then they must work with short focal lengths, what in turn produces aberrations.

Anyhow, the Rayleigh criterion is quite strict. Cameras with APS format, for example, can use $f / 22$ with scarce diffraction problems.

\section{Confusion circle and depth of field}

The circle of confusion ( $\mathrm{CoC}$ ) is a measurement of the blur circle produced by the lenses when the image is brought inside the camera. The further away from the image plane, the most blurred the scene is. However, there is a certain $\mathrm{CoC}$ diameter that can be considered "acceptably sharp". This is closely related with the depth of field.

When an object is focused, only one plane of the object is actually focused onto the film or sensor located at the image plane (Fig. 18). Any other object, or part of the same object, located in front (blue shape) or behind (red shape) this plane is not completely focused. The film or sensor intersects the cone of light rays originating in the object forming a disk whose diameter is larger as the distance between the object and the focusing plane is longer.

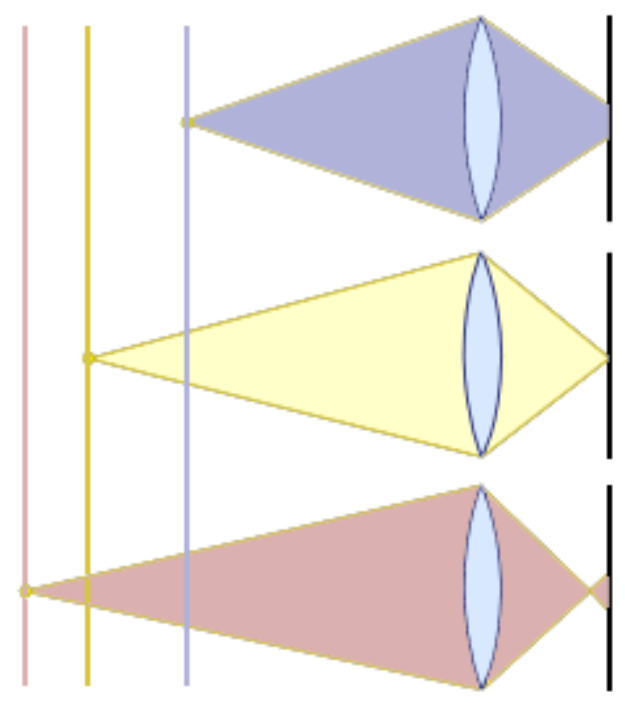

Figure 18. Confusion circles (right) derived from defocusing

The interesting point is to find out the border line between "focused" and "defocused". The problem is equivalent to finding the largest circle seen as a single point by the naked eye in an enlarged copy (CoC). The eye would detect features in any circle larger than this, hence defocusing would be detected. Under these circumstances, the limit would depend on:

- the size of the copy: a $40 \times 60$ blow-up would show more details than a $10 \times 15$ one

- the observation distance: more details are seen at $25 \mathrm{~cm}$ than at $1 \mathrm{~m}$

- the sensor size: for a picture of a given size, the image of a full-frame sensor requires less enlargement than the image of an APS-C sensor, simply because the sensor is larger

A simple formula to calculate the $\mathrm{CoC}$ is given by Zeiss [8]. For a blow-up copy of $24 \times 18 \mathrm{~cm}$ ( $30 \mathrm{~cm}$ diagonal), observed at $50 \mathrm{~cm}$, the formula is $C o C=d / 1730$ (it is customary to use $d / 1500$ instead). Here CoC is the diameter of the circle of confusion in $\mathrm{mm}$, and $d$ is the diagonal of the sensor in $\mathrm{mm}$ ( $45 \mathrm{~mm}$ for full frame and $27 \mathrm{~mm}$ for APS-C). The CoC diameter would be $0.029 \mathrm{~mm}$ in full frame and $0.018 \mathrm{~mm}$ in APS-C.

Three factors affect the depth of field (DoF) [9]:

- Focusing distance: the farther the focused object is, the longer the DoF. Focusing an object at $3 \mathrm{~m}$, keeping constant focal length and diaphragm aperture, the DoF may go from, say, $1.8 \mathrm{~m}$ to $5 \mathrm{~m}$. With the same parameters, an object at $0.5 \mathrm{~m}$ reduces the DoF to $0.4-0.7 \mathrm{~m}$.

- Focal length: the longer the objective focal length, the shorter the DoF. 
- Diaphragm aperture: the larger the aperture (lower $f \#$ ), the shorter the DoF. This is obvious if one considers that a closed diaphragm reduces the light cone, hence reducing the size of the circle of confusion, i.e., increasing the "focused" range (Fig. 19).

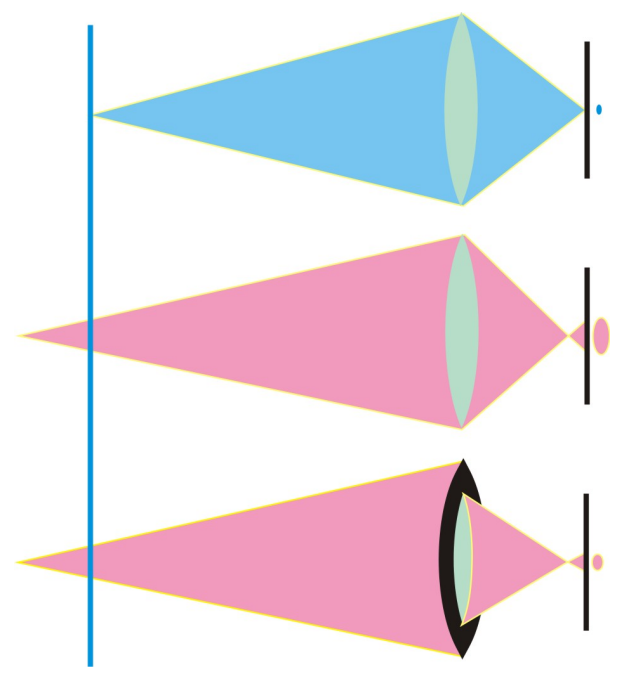

Figure 19. When closing the diaphragm, the circle of confusion of the images outside the focal plane is reduced

The so-called hyperfocal distance is the shortest distance at which the image of an object is neatly seen in a camera that is focusing a plane at the infinity as well.

\section{Aberrations}

Aberrations are deformations of the image derived from the non-ideality of the optical components. All optical systems feature aberrations to a larger or smaller extent. In photography, aberrations depend on the diaphragm aperture and the Field of View (FoV) angle or visual angle. Quite often dependences are nonlinear. The more luminous an objective is, and the shorter its focal length, the more difficult is to avoid and correct aberrations. Moreover, aberrations also increase with increasing sensor format. Table 3 summarizes the most relevant aberrations and their dependence on these parameters.

TABLE 3. Common aberrations and dependences

\begin{tabular}{|l||l|l||l||}
\cline { 2 - 4 } \multicolumn{1}{c|}{} & Diaphragm aperture & Field of View & Distance from the axis \\
\hline Spherical aberration & $\Phi^{3}$ & No dependence & No dependence \\
Coma & $\Phi^{2}$ & $($ FoV $)$ & D \\
Curvature & $\Phi$ & $(\text { FoV })^{2}$ & $D^{2}$ \\
Astigmatism & $\Phi$ & $(\text { FoV })^{2}$ & $D^{2}$ \\
Distortion (\%) & No dependence & $(\text { FoV })^{3}$ & $D^{2}$ \\
Chromatic Axial Aberration & $\Phi$ & No dependence & No dependence \\
Chromatic Lateral Aberration & No dependence & $1 /($ FoV $)$ & D \\
\hline \hline
\end{tabular}

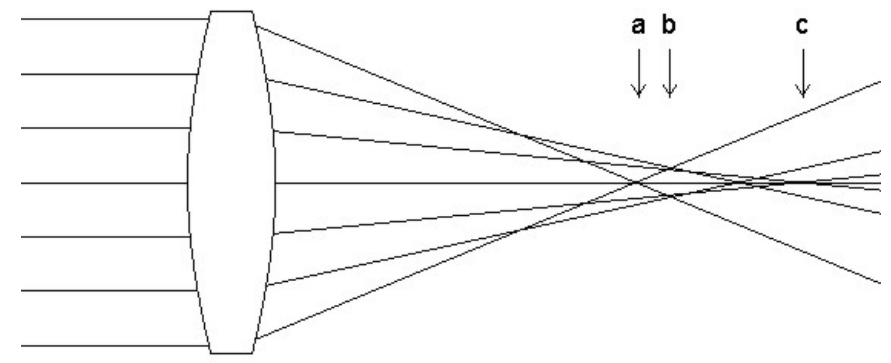

Figure 20. Spherical aberration and focus shift upon diaphragm variations 
As seen in the Table, aberrations are reduced when closing the diaphragm or reducing the FoV (zooming). On the contrary, wide-angle objectives increase significantly the spherical aberration and the distortion.

The spherical aberration is responsible for an effect called focus shift, consisting in a change in the focus distance when the diaphragm is modified. Figure 20 shows an example of spherical aberration. The outer rays are not focused on the same point as the inner rays (points $a$ and $c$ respectively). If we choose focusing at point $b$, and eventually we close the diaphragm, focusing moves to point $c$, thus defocusing the image.

Spherical aberration arises from the use of spherical lenses (it can be demonstrated that a spherical cap does NOT focus parallel rays to the same point). Therefore, this aberration can be corrected by using nonspherical, or aspheric, lenses. However, this may increase other aberrations, especially if the diaphragm is far from the lens.

Coma (or oblique spherical aberration) is produced by differences in refraction of light rays whose incidence angle is different in the curved surface -same as spherical aberration. In this case, light with larger incidence angle is tilted more than light impinging close to the lens axis (Fig. 21).

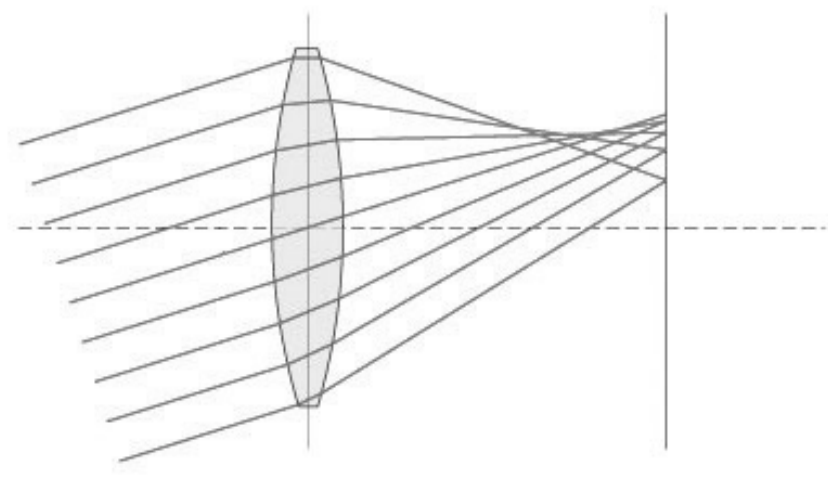

Figure 21. Coma or oblique spherical aberration

Other achromatic aberrations, like astigmatism and field curvature, arise from the flatness of the focal plane, since the actual focal plane is curved (the eye provides a better solution here).

Chromatic aberrations are related to the different wavelengths of the incoming light, and the fact that virtually no material has a constant refractive index with varying wavelength. Indeed, in most materials the refractive index is higher for shorter wavelengths (blue) and lower for longer wavelengths (red). As a consequence, the blue-violet spectral region is focused closer to the lens than the red region. The human eye is nearly free of achromatic aberrations, but it shows chromatic aberrations as well.

The most important are called axial and lateral chromatic aberrations (Fig. 22); these are conceptually equivalent to spherical aberration and coma respectively. Chromatic aberrations are corrected with achromatic doublets or triplets, sets of lenses that mutually compensate their chromatic aberrations.

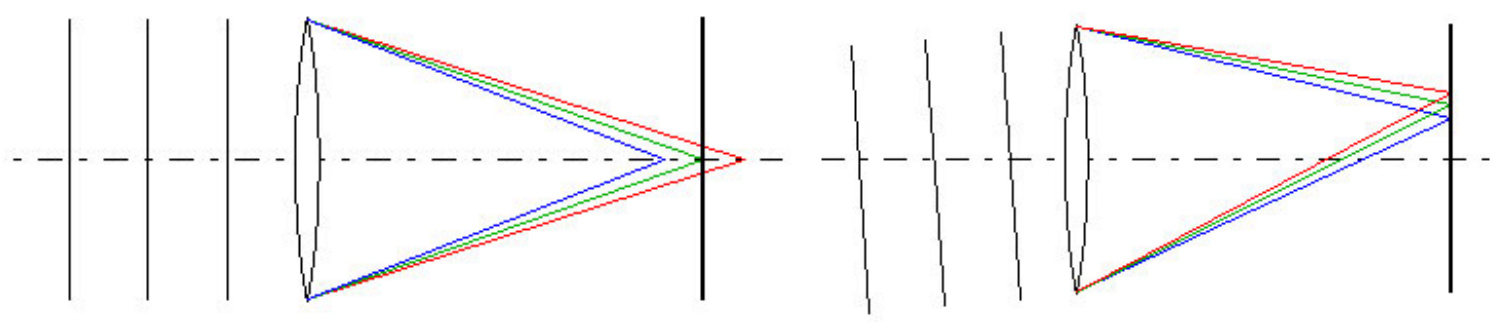

Figure 22. Chromatic axial and lateral aberrations

\section{Conclusions}

The first part of this course on digital photography has concentrated on the optical aspects of the cameras, their parameters and measurements, and their comparison with human vision. With this background, it is possible to deal with the second part, that is devoted entirely to optoelectronic features of the sensors, 
ÓPTICA PURA Y APLICADA

www.sedoptica.es

including a description and comparative study of the main commercial types of sensors, mechanisms of gathering and storing images, and color handling. 